\title{
An Evidence Review of Face Masks Against
} COVID-19

\author{
Jeremy Howard ${ }^{\mathrm{a}, \mathrm{c}, 1}$, Austin Huang ${ }^{\mathrm{b}}$, Zhiyuan $\mathrm{Li}^{\mathrm{k}}$, Zeynep Tufekci ${ }^{\mathrm{m}}$, Vladimir Zdimal ${ }^{\mathrm{e}}$, Helene-Mari van der Westhuizen ${ }^{\mathrm{f}, \mathrm{g}}$, Arne \\ von Delft ${ }^{0, g}$, Amy Price $^{\mathrm{n}}$, Lex Fridman ${ }^{\mathrm{d}}$, Lei-Han Tang ${ }^{\mathrm{i}, \mathrm{j}}$, Viola Tang ${ }^{\mathrm{l}}$, Gregory L. Watson ${ }^{\mathrm{h}}$, Christina E. Bax ${ }^{\mathrm{s}}$, Reshama Shaikh ${ }^{\mathrm{q}}$, \\ Frederik Questier ${ }^{r}$, Danny Hernandez ${ }^{\mathrm{p}}$, Larry F. Chu ${ }^{\mathrm{n}}$, Christina M. Ramirez ${ }^{\mathrm{h}}$, and Anne W. Rimoin ${ }^{\mathrm{t}}$
}

This manuscript was compiled on October 30, 2020

\begin{abstract}
The science around the use of masks by the general public to impede COVID-19 transmission is advancing rapidly. In this narrative review, we develop an analytical framework to examine mask usage, considering and synthesizing the relevant literature to inform multiple areas: population impact; transmission characteristics; source control; PPE; sociological considerations; and implementation considerations. A primary route of transmission of COVID-19 is via respiratory particles (droplets and aerosols), and is known to be transmissible from presymptomatic, paucisymptomatic and asymptomatic individuals. Reducing disease spread requires two things: limit contacts of infected individuals via physical distancing and other measures, and reduce the transmission probability per contact. The preponderance of evidence indicates that mask wearing reduces the transmissibility per contact by reducing transmission of infected aerosols and droplets in both laboratory and clinical contexts. Public mask wearing is most effective at reducing spread of the virus when compliance is high. Given the current shortages of medical masks we recommend the adoption of public cloth mask wearing, as an effective form of source control, in conjunction with existing hygiene, distancing, and contact tracing strategies. Because many respiratory particles become smaller due to evaporation, we recommend increasing focus on a previously overlooked aspect of mask usage: mask-wearing by infectious people ("source control") with benefits at the population-level, rather than only mask-wearing by susceptible people, such as health-care workers, with focus on individual outcomes. We recommend that public officials and governments strongly encourage the use of widespread face masks in public, including the use of appropriate regulation.
\end{abstract}

COVID-19 | SARS-CoV-2 | Masks | Pandemic

Policymakers need urgent guidance on the use of masks by the general population as a tool in combating SARS-CoV-2, the respiratory virus that causes COVID-19. Masks have been recommended as a potential tool to tackle the COVID-19 pandemic since the initial outbreak in China (1), although usage during the outbreak varied by time and province (2). Globally, countries are grappling with translating the evidence of public mask wearing to their contexts. These policies are being developed in a complex decision-making environment, with a novel pandemic, rapid generation of new research, and exponential growth in cases and deaths in many regions. There is currently a global shortage of N95/FFP2 respirators and surgical masks for use in hospitals. Simple cloth masks present a pragmatic solution for use by the public. This has been supported by most health bodies. We present an interdisciplinary narrative review of the literature on the role of face masks in reducing COVID-19 transmission in the

community.

\section{Background}

Wu Lien Teh's work to control the 1910 Manchurian Plague has been acclaimed as "a milestone in the systematic practice of epidemiological principles in disease control" (3), in which Wu identified the cloth mask as "the principal means of personal protection." Although Wu designed the cloth mask that was used through most of the world in the early 20th century, he pointed out that the airborne transmission of plague was known since the 13th century, and face coverings were recommended for protection from respiratory pandemics since the 14th century, recommendations that were still in place at the end of the 19th century (4). Wu reported on experiments that showed a cotton mask was effective at stopping airborne transmission, as well as on observational evidence of efficacy for healthcare workers. Masks have continued to be widely used to control transmission of respiratory infections in

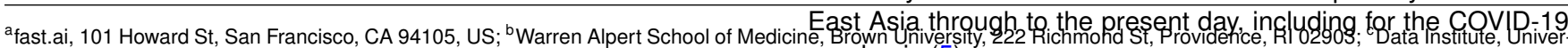

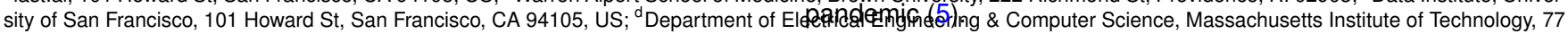
Massachusetts Ave, Cambridge, MA 02139; ' Institute of Chemical Process Fundamentals, Czech Academy of Sciences, Rozvojová 135, CZ-165 02 Praha 6, Czech Republic; ${ }^{f}$ Department of Primary Health Care Sciences, Woodstock Road, University of Oxford, OX2 6GG, United Kingdom; ${ }^{9}$ TB Proof, Cape Town, South Africa; ${ }^{\text {h Department of }}$ Biostatistics, UCLA Fielding School of Public Health, 650 Charles E Young Drive, Los Angeles, CA 90095; 'Department of Physics, Hong Kong Baptist University, Kowloon Tong, Hong Kong SAR, China; ${ }^{j}$ Complex Systems Division, Beijing Computational Science Research Center, Haidian, Beijing 100193 , China; ${ }^{\mathrm{k}}$ Center for Quantitative Biology, Peking University, Haidian, Beijing 100871, China; 'Department of Information Systems, Business Statistics and Operations Management, Hong Kong University of Science and Technology, Clear Water Bay, Kowloon, Hong Kong SAR, China; ${ }^{m}$ University of North Carolina at Chapel Hill; ${ }^{\mathrm{n}}$ School of Medicine Anesthesia Informatics and Media (AIM) Lab, Stanford University, 300 Pasteur Drive, Grant S268C, Stanford, CA 94305; ${ }^{\circ}$ School of Public Health and Family Medicine, University of Cape Town, Anzio Road, Observatory, 7925, South Africa; ${ }^{\mathrm{P} O p e n A I,} 3180$ 18th St, San Francisco, CA 94110; ${ }^{\mathrm{C}}$ Data Umbrella, 345 West 145 th St, New York, NY 10031 ; ${ }^{\mathrm{V}}$ Vrije Universiteit Brussel, Pleinlaan 2, 1050 Brussels, Belgium; ' University of Pennsylvania, 3400 Civic Center Blvd, Philadelphia, PA 19104; 'Department of Epidemiology, UCLA Fielding School of Public Health, 650 Charles E Young Drive, Los Angeles, CA 90095

Jeremy Howard prepared the initial literature list; Reshama Shaikh prepared the initial literature summaries; Frederik Questier conducted additional literature searches and summaries; Zhiyuan Li, Violet Tang, Lei-Han Tang, and Danny Hernandez did impact modeling; Zeynep Tufekci provided sociological research and analysis; Helene-Mari van der Westhuizen and Arne von Delft provided analysis of implementation considerations; Christina Bax provided review and feedback; All authors contributed to the writing.

${ }^{1}$ To whom correspondence should be addressed. E-mail: jphoward@usfca.edu 
In other parts of the world, however, mask usage in the community had fallen out of favor, until the impact of COVID-19 was felt throughout the world, when the discarded practice was rapidly reverted. By the end of June 2020 , nearly $90 \%$ of the global population lived in regions that had nearly universal mask use, or had laws requiring mask use in some public locations (6), and community mask use was recommended by nearly all major public health bodies, including the CDCs of United States, China, and Europe, and the World Health Organization. This is a radical change from the early days of the pandemic, when masks were infrequently recommended or used.

\section{Direct evidence of the efficacy of public mask wear- ing}

If there is strong direct evidence, either a suitably powered randomized controlled trial (RCT), or a suitably powered metaanalysis of RCTs, or a systematic review of unbiased observational studies that finds compelling evidence, then that would be sufficient for evaluating the efficacy of public mask wearing, at least in the contexts studied. Therefore, we start this review looking at these types of evidence.

A. Direct epidemiological evidence. Cochrane (7) and the World Health Organization (8) both point out that for population health measures we should not generally expect to be able to find controlled trials, due to logistical and ethical reasons, and should therefore instead seek a wider evidence base. This issue has been identified for studying community use of masks for COVID19 in particular (9). Therefore, we should not be surprised to find that there is no randomized controlled trial (RCT) for the impact of masks on community transmission of any respiratory infection in a pandemic.

Only one observational study has directly analyzed the impact of mask use in the community on COVID-19 transmission. The study looked at the reduction of secondary transmission of SARSCoV-2 in Beijing households by face mask use (10). It found that facemasks were $79 \%$ effective in preventing transmission, if they were used by all household members prior to symptoms occurring. The study did not look at the relative risk of different types of mask.

In a systematic review sponsored by the World Health Organization, Chu et al looked at physical distancing, face masks, and eye protection to prevent person-to-person transmission of SARSCoV-2 (11). They found that "face mask use could result in a large reduction in risk of infection." However, the review included only three studies of mask use outside healthcare settings, all of which were of SARS, not of SARS-CoV-2, and one of which was incorrectly categorized (it occurred in a hospital, but during family and friend visits), and one of which found that none of the households wearing masks had any infections, but was too under-powered to draw any conclusions (12). The remaining study found the use of masks was strongly protective, with a risk reduction of $70 \%$ for those that always wore a mask when going out (13), but it did not look at the impact of masks on transmission from the wearer. It is not known to what degree analysis of other coronaviruses can be applied to SARS-CoV-2. None of the studies looked at the relative risk of different types of mask.

There has been one controlled trial of mask use for influenza control in the general community (14). The study looked at Australian households, was not done during a pandemic, and was done without any enforcement of compliance (such as would be provided by a mask mandate). It found that "in an adjusted analysis of compliant subjects, masks as a group had protective efficacy in excess of $80 \%$ against clinical influenza-like illness." However, the authors noted that they "found compliance to be low, but compliance is affected by perception of risk. In a pandemic, we would expect compliance to improve." In compliant users, masks were highly effective at reducing transmission.

Overall, evidence from RCTs and observational studies are informative, but not compelling on their own. Both the Australian influenza RCT and the Beijing households observational trial found around $80 \%$ efficacy amongst compliant subjects, and the one SARS household study of sufficient power found $70 \%$ efficacy for protecting the wearer. However, we do not know if the results from influenza or SARS will correspond to results for SARS-CoV2 , and the single observational study of SARS-CoV-2 might not be replicated in other communities. None of the studies looked specifically at cloth masks.

\section{B. Reviews and RCTs of mask use for other respiratory ill-} nesses. A number of reviews have investigated masks during nonpandemic outbreaks of influenza and other respiratory diseases. However, it is not known to what degree these findings apply to pandemic SARS-CoV-2. When evaluating the available evidence for the impact of masks on community transmission, it is critical to clarify the setting of the research study (health care facility or community), whether masks are evaluated as source control or protection for the wearer or both, the respiratory illness being evaluated, and (for controlled trials) what control group was used.

A Cochrane review (15) on physical interventions to interrupt or reduce the spread of respiratory viruses included 67 studies that were randomized controlled trials and observational studies. It found that "overall masks were the best performing intervention across populations, settings and threats." There is a similar preprint review by the same lead author (16), in which only studies where mask wearing was tested as a stand-alone intervention were included, without combining it with hand hygiene and physical distancing, and excluding observational studies. That review concluded that "there was insufficient evidence to provide a recommendation on the use of facial barriers without other measures." Maclntyre (17) published a review evaluating masks as protective intervention for the community, protection for health workers, and as source control. The authors conclude that "community mask use by well people could be beneficial, particularly for COVID-19, where transmission may be pre-symptomatic. The studies of masks as source control also suggest a benefit, and may be important during the COVID-19 pandemic in universal community face mask use as well as in health care settings."

The Usher Institute incorporated laboratory as well as epidemiological evidence in their review (18), finding that "homemade masks worn by sick people can reduce virus transmission by mitigating aerosol dispersal. Homemade masks worn by sick people can also reduce transmission through droplets." One preprint systematic review (19) including epidemiological, theoretical, experimental, and clinical evidence, found that "face masks in a general population offered significant benefit in preventing the spread of respiratory viruses especially in the pandemic situation, but its utility is limited by inconsistent adherence to mask usage." On the other hand, a preprint systematic review that only included RCTs and observational studies (20) concluded based on the RCTs that there was only weak evidence for a small effect from mask use in the community, but that the RCTs often suffered from poor compliance and controls. It found that in observational studies the 
evidence in favour of wearing face masks was stronger.

Randomised control trial evidence that investigated the impact of masks on household transmission during influenza epidemics indicate potential benefit, although we should be careful of assuming these results will transfer to SARS-CoV-2. In particular, influenza has an $R_{0}$ (the basic reproduction number) of 1.4 (21) whereas SARS-CoV-2 has an $R_{0}$ of 2.4 or more (22). Suess et al conducted an RCT (23) that suggests household transmission of influenza can be reduced by the use of non-pharmaceutical interventions, namely the use of face masks and intensified hand hygiene, when implemented early and used diligently. Concerns about acceptability and tolerability of the interventions should not be a reason against their recommendation (23). Cowling et al (24) investigated hand hygiene and face masks in an RCT that seemed to prevent household transmission of influenza virus when implemented within 36 hours of index patient symptom onset. These findings suggest that non-pharmaceutical interventions are important for mitigation of pandemic and inter-pandemic influenza. RCT findings by Aiello et al (25) "suggest that face masks and hand hygiene may reduce respiratory illnesses in shared living settings and mitigate the impact of the influenza $A(\mathrm{H} 1 \mathrm{~N} 1)$ pandemic." A randomized intervention trial (26) found that "face masks and hand hygiene combined may reduce the rate of ILI [influenza-like illness] and confirmed influenza in community settings. These non-pharmaceutical measures should be recommended in crowded settings at the start of an influenza pandemic." The authors noted that their study "demonstrated a significant association between the combined use of face masks and hand hygiene and a substantially reduced incidence of ILI during a seasonal influenza outbreak. If masks and hand hygiene have similar impacts on primary incidence of infection with other seasonal and pandemic strains, particularly in crowded, community settings, then transmission of viruses between persons may be significantly decreased by these interventions."

Overall, direct evidence of the efficacy of mask use is supportive, but inconclusive. Since there are no randomized controlled trials, only one observational trial, and unclear evidence from other respiratory illnesses, we will need to look at a wider body of evidence.

\section{A framework for considering the evidence}

The standard RCT paradigm is well-suited to medical interventions in which a treatment has a measurable effect at the individual level and furthermore, interventions and their outcomes are independent across persons comprising a target population.

By contrast, the effect of masks on a pandemic is a populationlevel outcome where individual-level interventions have an aggregate effect on their community as a system. Consider, for instance, the impact of source control - its effect occurs to other individuals in the population, not the individual who implements the intervention by wearing a mask. This also underlies a common source of confusion - most RCT studies in the field examine masks as personal protective equipment (PPE) because efficacy can be measured in individuals to whom treatment is applied, i.e. "did the mask protect the person who wore it?" Even then, ethical issues prevent the availability of an unmasked control arm (27).

The lack of direct causal identifiability requires a more integrative systems view of efficacy. We need to consider first principles - transmission properties of the disease, controlled biophysical characterizations - alongside observational data, partially informative RCTs (primarily with respect to PPE), natural experiments
(28), and policy implementation considerations - a discursive synthesis of interdisciplinary lines of evidence which are disparate by necessity $(9,29)$.

The goal of such an analysis is to assess the potential benefits and risks, in order to inform policy and behavior. UNESCO states that "when human activities may lead to morally unacceptable harm that is scientifically plausible but uncertain, actions shall be taken to avoid or diminish that harm" (30). This is known as the "precautionary principle." The World Charter for Nature, which was adopted by the UN General Assembly in 1982, was the first international endorsement of the precautionary principle. It was implemented in an international treaty in the 1987 Montreal Protocol. The loss of life and economic destruction that has been seen already from COVID-19 are "morally unacceptable harms."

In order to identify whether public mask wearing is an appropriate policy, we need to consider the following questions, and assess based on their answers whether mask wearing is likely to diminish harm based on the precautionary principle:

a Population impact: What could the overall population-level impact of public mask wearing be?

b Transmission characteristics: Based on our understanding of virus transmission, what would be required for a mask to be effective?

c Source control: Do face masks decrease the number of people infected by an infectious mask wearer?

d PPE: Do face masks impact the probability of the wearer becoming infected themselves?

e Sociological considerations: can masks lead to unintended benefits or harm (for example risk compensation behaviour)?

f Implementation consideration: how can medical supply chains be maintained?

We will evaluate each consideration in turn.

\section{Population impact}

There are now over 100 countries that have implemented mask requirements (31), and many regions such as US states that have their own mask mandates. Most of these requirements were instituted after there was a shortage of medical masks, so results in these countries are likely to reflect the reality of what masks the public is able to access in practice during a pandemic. By analyzing the timing of pandemic spread and mask use, along with confounders such as population and geographic statistics, and timings of other policy interventions, it is possible to estimate the impact of mask use at a policy level. Here we look at studies based on this approach, as well as looking at estimated outcomes based on models, as part of a broad population impact analysis.

A. Ecological studies. Leffler et al (31) used a multiple regression approach, including a range of policy interventions and country and population characteristics, to infer the relationship between mask use and SARS-CoV-2 transmission. They found that transmission was 7.5 times higher in countries that did not have a mask mandate or universal mask use, a result similar to that found in an analogous study of fewer countries (32). Another study looked at the difference between US states with mask mandates versus those without, and found that the daily growth rate 
was 2.0 percentage points lower in states with mask mandates, estimating that the mandates had prevented 230,000 to 450,000 COVID-19 cases by May 22, 2020 (33).

The approach of Leffler et al was replicated by Goldman Sachs for both US and international regions, finding that face masks have a large reduction on infections and fatalities, and estimating a potential impact on US GDP of one trillion dollars if a nationwide mask mandate were implemented (34). Although between-region comparisons do not allow for direct causal attribution, they suggest mask wearing to be a low-risk measure with a potentially large positive impact.

A paper in the American Journal of Respiratory and Critical Care Medicine (35) which analyzed Google Trends, E-commerce, and case data, found that early public interest in face masks may be an independently important factor in controlling the COVID19 epidemic on a population scale. Abaluck et al (36) extend the between-country analyses from a cost perspective, estimating the marginal benefit per cloth mask worn to be in the range from US $\$ 3,000$ to US $\$ 6,000$.

A study of COVID-19 incidence in Hong Kong noted that face mask compliance was very high, at $95.7 \%$ to $97.2 \%$ across regions studied, and that COVID-19 clusters in recreational mask-off settings were significantly more common than that in workplace mask-on settings (37).

B. Modeling. At the national and global scale, effective local interventions are aggregated into epidemiological parameters of disease spread. The standard epidemiological measure of spread is known as the basic reproduction number $R_{0}$ which provides parameters for the average number of people infected by one person, in a susceptible population with no interventions. The goal of any related healthcare policy is to have an aggregate effect of reducing the effective reproduction number $R_{e}$ to below $1 . R_{e}$ is the average number of people infected by one person in a population in practice, including the impact of policies, behavior change, and already infected people.

Efficacy of face masks within local interventions would have an aggregate effect on the reproduction number of the epidemic. In this section we look at models that have attempted to estimate the possible magnitude of such an effect. The basic reproduction number $R_{0}$ is estimated to be in the range 2.4-3.9 (22).

Stutt et al (38) explain that it is impossible to get accurate experimental evidence for potential control interventions, but that this problem can be approached by using mathematical modelling tools to provide a framework to aid rational decision-making. They used two complementary modelling approaches to test the effectiveness of mask wearing. Their models show that mask use by the public could significantly reduce the rate of COVID-19 spread, prevent further disease waves and allow less stringent lock-down measures. The effect is greatest when $100 \%$ of the public wear face masks. They found that with a policy that all individuals must wear a mask all of the time, a median effective COVID-19 $R_{e}$ of below 1 could be reached, even with mask effectiveness of $50 \%$ (for $R_{0}=2.2$ ) or of $75 \%$ (for $R_{0}=4$ ).

Kai et al (39) presented two models for predicting the impact of universal mask wearing. Both models showed a significant impact under (near) universal masking when at least $80 \%$ of a population is wearing masks, versus minimal impact when only $50 \%$ or less of the population is wearing masks. Their models estimated that $80-90 \%$ masking would eventually eliminate the disease. They also looked at an empirical dataset, finding a very strong correlation between early universal masking and successful suppression of daily case growth rates and/or reduction from peak daily case growth rates, as predicted by their theoretical simulations.

Tian et al developed a simple transmission model that incorporated mask wearing and mask efficacy as a factor in the model (40). They estimate reductions in the effective reproduction number, $R_{e}$, which is the number of people on average each patient infects following interventions and behavior changes, under common intervention measures. For wearing masks, they found that wearing masks reduces $R_{e}$ by a factor $(1-m p)^{2}$, where $m$ is the efficacy of trapping viral particles inside the mask, and $p$ is the percentage of the population that wears masks. When combined with contact tracing, the two effects multiply. The paper notes that an important issue not treated explicitly is the role played by asymptomatic carriers of the virus. In addition, if adherence is socioeconomically, demographically or geographically clustered, the mass action model may overestimate the impact. This is a limitation that could apply to all the models discussed in this review.

Under the Tian et al model, the largest effects are seen when $R_{0}$ is high, since the factor discussed above is a multiplier of $R_{0}$. Therefore, we will consider a conservative assessment applied to an assumed $R_{0}$ of 2.4, which is at the low end of the range presented above, and also supported by other studies (41). With $50 \%$ mask usage and $50 \%$ mask efficacy level, $(1-m p)^{2}=0.56$. Thus an $R_{0}$ of 2.4 is reduced to an $R_{e}$ of $2.4 \times 0.56=1.34$, a huge impact rendering spread comparable to the reproduction number of seasonal influenza. To put this in perspective, 100 cases at the start of a month become 584 cases by the month's end $\left(R_{e}=1.34\right)$ under these assumptions, versus 31,280 cases $\left(R_{e}=2.4\right)$ if masks are not used. Such a slowdown in caseload protects healthcare capacity and renders a local epidemic amenable to contact tracing interventions that could eliminate the spread entirely. At the high end of the range, an $R_{0}$ of 3.9 will become an $R_{e}$ of 2.2 under the same assumptions.

A full range of efficacy $m$ and adherence $p$ based on an $R_{0}$ of 2.4 is shown with the resulting $R_{e}$ in Figure 1, illustrating regimes in which growth is dramatically reduced $\left(R_{e}<1\right)$ as well as pessimistic regimes (e.g. due to poor implementation or population compliance) that nonetheless result in a beneficial effect in suppressing the exponential growth of the pandemic. For different values of $R_{0}$, the image would be identical, with just the color bar scale varying linearly with the change in $R_{0}$.

Ngonghala et al use a similar approach, covering a wider variety of interventions, and completing numerous numerical simulations (42). They find that "high use of face-masks in public could lead to COVID-19 elimination," and that "combining face-masks and social-distancing is more effective in COVID-19 control." Yan et al (43) provide an additional example of an incremental impact assessment of respiratory protective devices using an augmented variant of a traditional SIR model in the context of influenza with N95 respirators. They showed that a sufficiently high adherence rate ( $~ 80 \%$ of the population) resulted in the elimination of the outbreak with most respiratory protective devices. Fisman et al (44) used a next-generation matrix approach to estimate the conditions under which masks would reduce the reproduction number of COVID-19 under a threshold of 1 . Their results supported the other models discussed in this section, finding that masks, even with sub-optimal efficacy in both prevention of acquisition and transmission of infection, could substantially decrease the reproduction number $R_{e}$ if widely used.

The models presented in this section are only as accurate as their assumptions and parameters. Kai et al (39) did compare their 


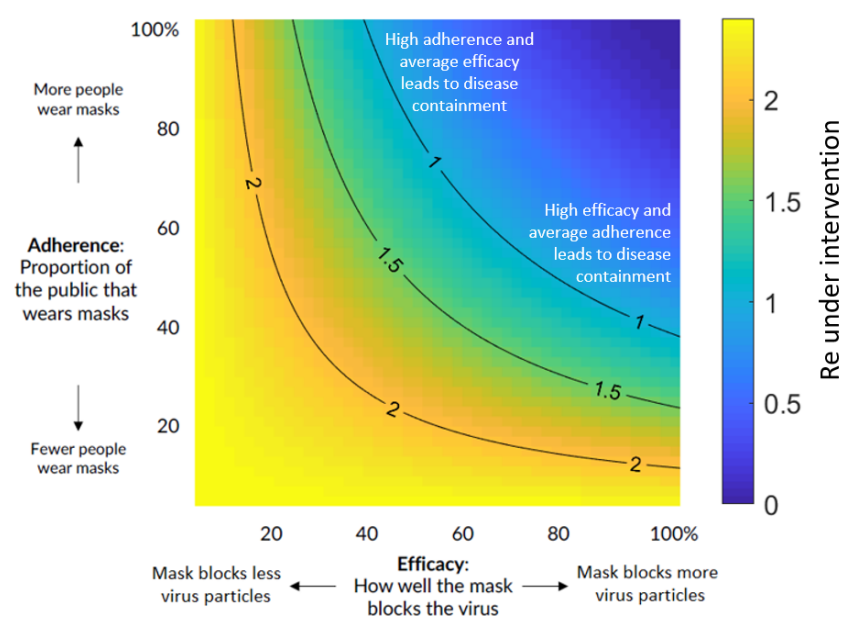

Fig. 1. Impact of public mask wearing under the full range of mask adherence and efficacy scenarios. The color indicates the resulting reproduction number $R_{e}$ from an initial $R_{0}$ of 2.4 (41). Blue area is what is needed to slow the spread of COVID-19. Each black line represents a specific disease transmission level with the effective reproduction number Re indicated. An $R_{e}$ below 1 , if sustained, will lead to the outbreak ending.

model's predictions with empirical results, and overall the models presented here are consistent with each other, and consistent with the empirical findings in the previous section. However, simulation, SEIR, and similar models are simplifications of the real world, which is much more complex, and cannot fully model all of the interactions and drivers of results in practice.

Overall, population-level studies of the impact of wearing masks suggest that mask use may have been an important driver of differences in SARS-CoV-2 outcomes in different regions. These outcomes are in line with models that predict substantial population level impacts of widespread mask use.

\section{Transmission characteristics}

We have seen that the efficacy of public mask wearing is largely supported by epidemiological and ecological data, as well as models. This could be due to masks filtering virus from an infected wearer, or protecting the wearer from infectious people around them, or both. In order to understand who should wear what kind of mask, and in what situations, we need an understanding of virus transmission.

Some COVID-19 patients are asymptomatic, and nearly all have a pre-symptomatic incubation period ranging from 2 to 15 days, with a median length of 5.1 days (45). Patients may be most infectious when symptoms are mildest or not present (4650). This characteristic differentiates SARS-CoV-2 (COVID-19) from SARS-CoV, as replication is activated early in the upper respiratory tract (51). A study of temporal dynamics inferred that infectiousness started from 2.3 days before symptom onset and peaked at 0.7 days before symptom onset (22).

High viral titers of SARS-CoV-2 are reported in the saliva of COVID-19 patients. These titers have been highest at time of patient presentation and viral levels are just as high in asymptomatic or presymptomatic patients, and occur predominantly in the upper respiratory tract (URT) $(47,51)$. Asymptomatic people seem to account for approximately $40 \%$ to $45 \%$ of SARS-CoV-2 infections (52). An analysis of SARS-CoV-2 viral load by patient age showed that viral loads of SARS-CoV-2 in children are similar to adults (53). Another paper showed no significant difference in saliva loads between mildly symptomatic and asymptomatic children. These findings support the contention that everyone, adults and children, should wear masks (54).

A consequence of these disease characteristics is that any successful policy intervention must properly address transmission due to infectious patients that display few or no symptoms and may not realize that they are infected. Because people with symptoms, including coughing and sneezing, are generally expected to stay home, our focus will be on other transmission vectors: speaking, breathing, and contact.

This topic has been subject to added confusion due to debates about whether these particles should be referred to as droplets or aerosols, with implications about their ability to remain suspended in air over time (55)(56). Inconsistent use of terminology about respiratory particles that can transmit this disease has led to confusion for scientists, the public health community, and the general public. For this paper, we adopt the definition by Milton (56) that incorporates findings from modern aerosol physics which suggest that particles much larger than the $5 \mu \mathrm{m}$ boundary (a number that is sometimes cited by public health authorities as a droplet/aerosol cut-off) can remain suspended in air for many minutes or more, can waft around and, of significant consequence for public health implications for this pandemic, accumulate depending on currents of air and ventilation status of the environment (56). We will thus refer to these respiratory emissions as "respiratory particles" with the understanding that these include particles that are transmitted through the air in a manner beyond the "ballistic trajectories" traditionally assumed of respiratory droplets and thus, in face, include aerosols that can remain suspended in the air (56). While determining an exact number is not necessary for purposes of this review, according to latest research informed by modern aerosol physics, $100 \mu \mathrm{m}$ is considered the boundary between aerosols and droplets (56).

Normal speaking produces thousands of oral fluid particles (aerosols and droplets) between $1 \mu \mathrm{m}$ to $500 \mu \mathrm{m}$ (57), which can harbor respiratory pathogens, including SARS-CoV-2 (58). Many of these emissions will then evaporate and turn into aerosolized particles that are 3 to 5 -fold smaller, and can float for ten minutes or more in the air (58-60). Speech is known to emit up to an order of magnitude more particles than breathing $(55,61,62)$.

A recent analysis has found that transmission through talking may be a key vector (63), with louder speech creating increasing quantities and sizes of particles, and a small fraction of individuals behaving as speech superemitters, releasing an order of magnitude more aerosols than their peers (57). Vuorinen concluded, with high level of certainty, that a major part of particles of respiratory origin stay airborne for a long enough time for them to be inhaled. They noted that the number of particles produced by speaking is significant especially as it is normally done continuously over a longer period of time (64). Prather et al stated that aerosol transmission of viruses must be acknowledged as a key factor leading to the spread of infectious respiratory diseases, and that SARS-CoV-2 is silently spreading in aerosols exhaled by highly contagious infected individuals with no symptoms (65). They noted that masks provide a critical barrier. The site of inhalation is also effected by the size of these particles, with smallest particles ( $\leq 5 \mathrm{tm}$ ) able to reach into the respiratory bronchioles and alveoli in the lungs and medium-sized ones ((up to $1015 \mathrm{tgm}$ ) able to deposit in the "the trachea and large intrathoracic airways." (56) 
Aerosolized transmission dynamics are pathogen-specific, due to pathogen-specific peak shedding and inactivation rates $(66,67)$. Studies suggest that vibration of the vocal folds contributes more to particle atomization and the production of particles that carry microorganisms (66). SARS-CoV-2 is present in exhaled breath (68), but it is not known to what degree they are responsible for transmission. A study of influenza suggests that vocalization might be critical for creation of infection breath particles (69).

The ability of masks to filter particles depends on the particle size and trajectory, with smaller floating aerosols more challenging to filter than larger particles with momentum (70). Because speech produces more particles containing the SARS-CoV2 virus, and because transmission of SARS-CoV-2 without symptoms is associated with upper respiratory tract (URT) shedding, where particles formed through vocalization are likely to contain the virus, we should be particularly cognisant of the role of speech particles in transmission (63). Speech particles lose their momentum and become much smaller shortly after ejection, which is likely to make them easier to filter as source control (as egress at the wearer) than as PPE (at ingress to an susceptible person). We will look at source control and PPE efficacy in turn.

\section{Source control}

In this section, we study whether a face mask (particularly cloth or other unfitted masks) is likely to decrease the number of people infected by an infectious mask wearer. The use of mask-wearing by potentially infectious people is known as "source control."

There are two main ways to physically test a mask: 1) have someone wearing it vocalize, such as breathe, talk or cough or; 2) synthetically simulate these actions using a spray mechanism, such as a nebulizer. Because human actions are complex and hard to simulate correctly, the first approach is preferred where possible. There are, in turn, two ways to analyze the results of this approach: 1) directly or indirectly measure the amount of respiratory particles of differing sizes, or; 2) measure the amount of infectious particles.

A. Human studies: Infectious particles. There are currently no studies that measure the impact of any kind of mask on the amount of infectious SARS-CoV-2 particles from human actions. Other infections, however, have been studied. One of the most relevant papers (71) is one that compares the efficacy of surgical masks for source control for seasonal coronaviruses (NL63, OC43, 229E and HKU1), influenza, and rhinovirus. With ten participants, the masks were effective at blocking coronavirus particles of all sizes for every subject. However, masks were far less effective at blocking rhinovirus particles of any size, or of blocking small influenza particles. The results suggest that masks may have a significant role in source control for the current coronavirus outbreak. The study did not use COVID-19 patients, and it is not yet known whether SARS-CoV-2 behaves the same as these seasonal coronaviruses, which are of the same family.

In a pair of studies from 1962 and 1975, a portable isolation box was attached to an Andersen Sampler and used to measure orally expelled bacterial contaminants before and after masking. In one study, during talking, unmasked subjects expelled more than 5,000 contaminants per 5 cubic feet; $7.2 \%$ of the contaminants were associated with particles less than $4 \mu \mathrm{m}$ in diameter (72). Cloth masked subjects expelled an average of 19 contaminants per 5 cubic feet; $63 \%$ were less than $4 \mu \mathrm{m}$ in diameter. So overall, over $99 \%$ of contaminants were filtered. The second study used the same experimental setup, but studied a wider range of mask designs, including a 4-ply cotton mask. For each mask design, over $97 \%$ contaminant filtration was observed (73).

Johnson et al (74) found that no influenza could be detected by RT-PCR on sample plates at $20 \mathrm{~cm}$ distance of coughing patients wearing masks, while it was detectable without mask for 7 of the 9 patients. Milton et al (75) found surgical masks produced a 3.4 $(95 \% \mathrm{Cl} 1.8$ to 6.3$)$ fold reduction in viral copies in exhaled breath by 37 influenza patients. Vanden Driessche et al (76) used an improved sampling method based on a controlled human aerosol model. By sampling a homogeneous mix of all the air around the patient, the authors could also detect any aerosol that might leak around the edges of the mask. Among their 6 cystic fibrosis patients producing infected aerosol particles while coughing, the airborne Pseudomonas aeruginosa load was reduced by $88 \%$ when wearing a surgical mask compared with no mask. Wood et al (77) found for their 14 cystic fibrosis patients with high viable aerosol production during coughing, a reduction in aerosol Pseudomonas aeruginosa concentration at 2 meters from the source by using a N95 mask (94\% reduction, $\mathrm{P}<0.001)$, or surgical mask (94\%, $\mathrm{P}<0.001)$. Stockwell et al (78) confirmed in a similar Pseudomonas aeruginosa aerosol cough study that surgical masks are effective as source control. One study (79) found surgical masks to decrease transmission of tuberculosis by $56 \%$ when used as source control and measuring differences in guinea pig tuberculosis infections, and another found similar results for SARS-CoV-2 infections in hamsters, using a "mask curtain" (80).

Multiple simulation studies show the filtration effects of cloth masks relative to surgical masks. Generally available household materials had between a $58 \%$ and $94 \%$ filtration rate for $1 \mu \mathrm{m}$ bacteria particles whereas surgical masks filtered $96 \%$ of those particles (81). A tea cloth mask was found to filter $60 \%$ of particles between $0.02 \mu \mathrm{m}$ to $1 \mu \mathrm{m}$, where surgical masks filtered $75 \%$ (82). Simulation studies generally use a $30 \mathrm{~L} / \mathrm{min}$ or higher challenge aerosol, which is around about 3 to 6 times the ventilation of a human at rest or doing light work (81). As a result, simulation studies may underestimate the efficacy of the use of unfitted masks in the community in practice.

B. Human studies: Aerosol and Droplet filtration. Anfinrud et al (63) used laser light-scattering to sensitively detect the emission of particles of various sizes (including aerosols) while speaking. Their analysis showed that visible particles "expelled" in a forward direction with a homemade mask consisting of a washcloth attached with two rubber bands around the head remained very close to background levels in a laser scattering chamber while significant levels were expelled when speaking without a mask.

There are no studies that have directly measured the filtration of smaller or lateral particles in this setting, although using Schlieren imaging it has been shown that all kinds of masks greatly limit the spread of the emission cloud (83), consistent with a fluid dynamic simulation that estimated this filtration level at $90 \%$ (84). Another study used a manikin and visible smoke to simulate coughing, and found that a stitched cloth mask was the most effective of the tested designs at source control, reducing the jet distance in all directions from 8 feet (with no mask) to 2.5 inches (85).

One possible benefit of masks for source control is that they can reduce surface transmission, by avoiding droplets settling on surfaces that may be touched by a susceptible person. However, contact through surfaces is not believed to be the main way SARSCoV-2 spreads (86), and the risk of transmission through surfaces 
may be small (87).

In summary, there is laboratory-based evidence that household masks have filtration capacity in the relevant particle size range, as well as efficacy in blocking aerosols and droplets from the wearer (71). That is, these masks help people keep their emissions to themselves. A consideration is that face masks with valves do not capture respiratory particles as efficiently, bypassing the filtration mechanism, and therefore offer less source control (88).

\section{PPE}

In this section, we study whether a face mask is likely to decrease the chance of a potentially susceptible mask wearer becoming infected. The use of mask-wearing by potentially susceptible people is known as "Personal Protective Equipment (PPE)." Protection of the wearer is more challenging than source control, since the particles of interest are smaller. It is also much harder to directly test mask efficacy for PPE using a human subject, so simulations must be used instead. Masks can be made of different materials and designs (70) which influence their filtering capability.

There are two considerations when looking at efficacy: 1) the filtration of the material; 2) the fit of the design. There are many standards around the world for both of these issues, such as the U.S. National Institute for Occupational Safety and Health (NIOSH) N95 classification. The 95 designation means that when subjected to testing, the respirator blocks at least 95 percent of very small ( 0.3 micron) test particles. $\mathrm{NIOSH}$ tests at flow rates of $85 \mathrm{~L} / \mathrm{min}$, simulating a high work rate, which is an order of magnitude higher than rest or low intensity breathing. These are designed to be tests of the worst-case (i.e., it produces maximum filter penetration) because the test conditions are the most severe that are likely to be encountered in a work environment (89). These tests use particles that are much smaller than viruscarrying emissions, at much higher flow rates than normally seen in community settings, which means that masks that do not meet this standard may be effective as PPE in the community. The machines used for these studies are specifically designed for looking at respirators that hold their shape which are glued or attached with beeswax firmly to the testing plate. Flexible masks such as cloth and surgical masks can get pulled into the hole in the testing plate, which makes it a less suitable testing method for these designs.

A study of filtration using the NIOSH approach (90), but with $78 \mathrm{~nm}$ particles, was used as the basis for a table in World Health Organization's "Advice on the use of masks in the context of COVID-19" (91). There was over 90\% penetration for all cotton masks and handkerchiefs, and $50 \%-60 \%$ penetration for surgical masks and non-woven non-medical masks. Zhao et al used a similar approach, but at a lower $32 \mathrm{~L} / \mathrm{min}$ (which is still 3-6 times higher than human ventilation during light work) (92). They also tested materials after creating a triboelectric effect by rubbing the material with a latex glove for thirty seconds, finding that polyester achieved a quality factor $(\mathrm{Q})$ of $40 \mathrm{kP} / \mathrm{a}$, nearly ten times higher than a surgical mask. Without triboelectric charging it achieved a $Q$ of 6.8 , which was similar to a cotton t-shirt. They concluded that cotton, polyester, and polypropylene multilayered structures can meet or even exceed the efficiency of materials used in some medical face masks. However, it depends on the details of the material and treatment.

One recent study looked at the aerosol filtration efficiency of common fabrics used in respiratory cloth masks, finding that effi- cacy varied widely, from $12 \%$ to $99.9 \%$, at flow rates lower than at-rest respiration (93). Many materials had $\geq 96 \%$ filtration efficacy for particles $>0.3$ microns, including 600 TPI cotton, cotton quilt, and cotton layered with chiffon, silk, or flannel. A combination of materials was more effective than the materials on their own. These findings support studies reported in 1926 by Wu Lien Teh, which described that a silk face covering with flannel added over the mouth and nose was highly effective against pneumonic plague (4).

There are many designs of cloth masks, with widely varying levels of fit. There have been few tests of different designs. A simple mask cut from a t-shirt achieved a fit score of 67 , offering substantial protection from the challenge aerosol and showing good fit with minimal leakage (94). One study looked at unfitted surgical masks, and used three rubber bands and a paper clip to improve their fit (95). All eleven subjects in the test passed the N95 fit test using this approach. Wu Lien Teh noted that a rubber support could provide good fit, although he recommended that a silk covering for the whole head (and flannel sewed over nose and mouth areas), with holes for the eyes, tucked into the shirt, is a more comfortable approach that can provide good protection for a whole day (4).

Research focused on aerosol exposure has found all types of masks are at least somewhat effective at protecting the wearer. Van der Sande et al (96) found that "all types of masks reduced aerosol exposure, relatively stable over time, unaffected by duration of wear or type of activity," and concluded that "any type of general mask use is likely to decrease viral exposure and infection risk on a population level, despite imperfect fit and imperfect adherence" (97).

The review from Chu et al (11) included three observational studies of face mask use for SARS-CoV-2 in healthcare environments, all showing a risk ratio of 0.03 to 0.04 . However these studies were given a much lower weight in the review than studies of MERS and SARS, and the overall risk ratio for mask use in health care was estimated at 0.30 .

One of the most frequently mentioned,but misinterpreted, papers evaluating cloth masks as PPE for healthcare workers is one from Maclntyre et al (27). The study compared a "surgical mask" group which received 2 new masks per day, to a "cloth mask" group that received 5 masks for the entire 4 week period and were required to wear the masks all day, to a "control group" which used masks in compliance with existing hospital protocols, which the authors describe as a "very high level of mask use." There was "no mask" control group because it was deemed "unethical." The study does not inform policy pertaining to public mask wearing as compared to the absence of masks in a community setting. They found that regular supply of new surgical masks each day had significantly lower infection of rhinovirus than the group that wore a limited supply of cloth masks, consistent with other studies that show surgical masks provide poor filtration for rhinovirus, compared to seasonal coronaviruses (71).

Most of the research of masks as health worker PPE focuses on influenza, though it is not yet known to what extent findings from influenza studies apply to COVID-19 filtration. Wilkes et al (98) found that "filtration performance of pleated hydrophobic membrane filters was demonstrated to be markedly greater than that of electrostatic filters." A meta-analysis of N95 respirators compared to surgical masks (99) found "the use of N95 respirators compared with surgical masks is not associated with a lower risk of laboratory-confirmed influenza." Radonovich et al (100) found 
in an outpatient setting that "use of N95 respirators, compared with medical masks in the outpatient setting resulted in no significant difference in the rates of laboratory-confirmed influenza."

One possible additional benefit of masks as PPE is that it does not allow hands to directly touch the nose and mouth, which may be a transmission vector. The lipid barrier that protects viruses is destroyed within five minutes of touching the hands (101), and wearing a mask during that period could be protective. However, there are no case reports or laboratory evidence to suggest that touching the mask can cause infection.

Overall, it appears that cloth face covers can provide good fit and filtration for PPE in some community contexts, but results will vary depending on material and design, the way they are used, and the setting in which they are used.

\section{Sociological considerations}

Some of the concerns about public mask wearing have not been around primary evidence for the efficacy of source control, but concerns about how they will be used.

A. Risk compensation behavior. One concern around public health messaging promoting the use of face-covering has been that members of the public may use risk compensation behavior. This involves fear that public would neglect other measures like physical distancing and hand hygiene, based on overvaluing the protection a mask may offer due to an exaggerated or false sense of security (102). Similar arguments have previously been made for HIV prevention strategies $(103,104)$, motorcycle helmet laws (105), seat-belts (106) and alpine skiing helmets (107). However, contrary to predictions, risk compensation behaviors have not been significant at a population level, being out-weighed by increased safety in each case $(106,108-111)$. These findings strongly suggest that, instead of withholding a preventative tool accompanying it with accurate messaging that combines different preventative measures would display trust in the general public's ability to act responsibly and empower citizens. Polling and observational data from the COVID-19 pandemic has shown mask wearing to be positively correlated with other preventative measure, including hand hygiene $(112,113)$ physical distancing $(112,113)$ and reduced face touching (114). Three preprint papers reporting observational data suggests that masks may be a cue for others to keep a wider physical distance. (115-117)

\section{B. Managing the stigma associated with wearing a mask.} Stigma is a powerful force in human societies, and many illnesses come with stigma for the sick as well as fear of them. Managing the stigma is an important part of the process of controlling epidemics (118). Tuberculosis is an example of an illness where masks are used as source control, but became a public label associated with the disease. Many sick people are reluctant to wear a mask if it identifies them as sick,in an effort to avoid the stigma of illness $(119,120)$. Some health authorities have recommended wearing masks for COVID-19 only if people are sick; however, reports of people wearing masks being attacked, shunned and stigmatized have also been observed (121). In many countries, minorities suffer additional stigma and assumptions of criminality (122). Black people in the United States have reportedly been reluctant to wear masks in public during this pandemic for fear of being mistaken as criminals $(123,124)$. Even if it were possible to encourage only infected people to wear masks, it is not always possible for many people to know for sure if they are infected or not (125). Thus, it may not even be possible to have sick people alone wear masks, due to stigma, employer restrictions, or simple lack of knowledge of one's status without mask-wearing becoming universal policy.

C. Creating new symbolism around wearing a mask. Ritual and solidarity are important in human societies and can combine with visible signals to shape new societal behaviors $(126,127)$. Universal mask wearing could serve as a visible signal and reminder of the pandemic. Signaling participation in health behaviors by wearing a mask as well as visible enforcement can increase compliance with public mask wearing, but also other important preventative behaviors (128). Historically epidemics are a time of fear, confusion and helplessness $(129,130)$. Maskwearing, and even mask-making or distribution, can provide feelings of empowerment and self-efficacy (131). Health is a form of public good in that everyone else's health behaviors improve the health odds of everyone else $(132,133)$. This can make masks symbols of altruism and solidarity (134). Viewing masks as a social practice, governed by sociocultural norms, instead of a medical intervention, has also been proposed to enhance longer-term uptake (135).

While the focus of this article is on preventing the spread of COVID-19 through public mask wearing, many countries face concurrent epidemics of contagious respiratory diseases like tuberculosis and influenza. Face covering has been shown to also reduce the transmission of tuberculosis (79). Similarly, influenza transmission in the community declined by $44 \%$ in Hong Kong after the implementation of changes in population behaviors, including social distancing and increased mask wearing, enforced in most stores, during the COVID-19 outbreak (5).

\section{Implementation considerations}

Globally, health authorities have followed different trajectories in recommendations around the use of face masks by the public. In China, Taiwan, Japan and South Korea, face masks were utilized from the start of the pandemic (2). Other countries, like Czechia and Thailand, were early adopters in a global shift towards recommending cloth masks. We present considerations for the translation of evidence about public mask wearing to diverse countries across the globe, outside of the parameters of a controlled research setting.

\section{A. Supply chain management of N95 respirators and surgi- cal masks. There has been a global shortage of protective equip- ment for health workers, with health workers falling ill and dy- ing of COVID-19 (136). N95 respirators are recommended for health workers conducting aerosol-generating procedures during clinical care of COVID-19 patients, while surgical masks are rec- ommended otherwise (137). Strategies to manage the shortage of PPE have included sterilization and re-use of respirators, and appeals to the public to reduce their use of medical masks (138). There were early concerns that public messaging encouraging mask use will deplete critical supplies. Some regions, like South Korea and Taiwan, have combined recommendations for the pub- lic to use surgical masks with rapidly increasing production of sur- gical masks, whilst in other regions cloth masks are promoted as alternative to surgical masks as source control. Cloth masks of- fer additional sustainability benefits through re-use, thus limiting costs and reducing environmental waste.}


There is some literature suggesting that face shields could provide additional eye-protection along with better visibility of facial expressions and fewer obstacles for communities, such as people who rely on lip-reading for communication (139). However, face shields alone have a large escape through brow and down jets (83), which may make them less effective for source control and this remains an open research question.

B. Mandatory mask wearing. Ensuring compliance with nonpharmaceutical interventions can be challenging, but likely rapidly increases during a pandemic (140). Perceptions of risk play an important role in mask use (14). Telephone surveys during the SARS-CoV-2 outbreak in Hong Kong reported enhanced adherence to public mask wearing as the pandemic progressed over three weeks, with $74.5 \%$ self reported mask wearing when going out increasing to $97.5 \%$, without mandatory requirements (5). Similar surveys reported face mask use in Hong Kong during the SARS outbreak in 2003 as $79 \%$ (141), and approximately $10 \%$ during the influenza $A(H 1 N 1)$ pandemic in 2009 (142). This suggests that the public have enhanced awareness of their risk, and that they display higher adherence levels to prevention strategies than during other epidemics. During the COVID-19 pandemic, many countries have utilised mask mandates as implementation strategy. In Germany, implementing a mask mandate led to welldocumented, widespread uptake in the use of masks. (112) A preregistered experiment $(n=925)$ further showed that "a voluntary policy would likely lead to insufficient compliance, would be perceived as less fair, and could intensify stigmatization. A mandatory policy appears to be an effective, fair, and socially responsible solution to curb transmissions of airborne viruses." Although the use of mandates has been a polarising measure, it appears to be highly effective in shaping new societal norms.

Modelling suggests $(39,40)$ that population level compliance with public mask wearing of $70 \%$ combined with contact tracing would be critical to halt epidemic growth. Population level uptake of an intervention to benefit the whole population is similar to vaccinations. A common policy response to this conundrum is to ensure compliance by using laws and regulations, such as widespread state laws in the US which require vaccinations to attend school. Research shows that the strength of the mandate to vaccinate greatly influences compliance rates for vaccines and that policies that set a higher bar for vaccine exemptions result in higher vaccination rates (143). The same approach is now being used in many jurisdictions to increase mask wearing compliance, by mandating mask use in a variety of settings (such as public transportation or grocery stores or even at all times outside the home). Population analysis suggests that these laws are effective at increasing compliance and slowing the spread of COVID-19 $(31,33,34)$.

\section{Further research}

There are many important issues that need to be addressed. In this section, we suggest further research directions.

There is a need to understand how masks can be used throughout the day, by both children (at school) (54) and adults (at work). In a study of the effect of mask use on household transmission of SARS-CoV-2, masks were found to be highly effective, including children, and the secondary attack rate for children was found to be only half that of adults. However, the impact of masks on children was not compared to adults (10). A commentary stated that "Face masks are an effective, practical, nonphar- maceutical intervention that would reduce the spread of influenza among school children, while keeping schools open," but noted that "the effectiveness of this intervention strategy is highly dependent on compliance" (144). Some researchers have proposed that face shields may be appropriate in some environments, (139) but it has not been well studied. Research on the efficacy of face shields, including in combination with masks, is needed, along with research into the efficacy of masks with transparent windows for the mouth.

The impact of using masks to control transmission in the workplace has not been well studied. One issue that impacts both school and work usage is that over a full day's use masks may become wet, or dirty. A study of mask use in health care settings found that "respiratory pathogens on the outer surface of the used medical masks may result in self-contamination," and noted that "the risk is higher with longer duration of mask use ( $>6 \mathrm{~h})$ and with higher rates of clinical contact" (145). Further research is needed to clarify these issues. In the meantime, most health bodies recommend replacing dirty or wet masks with clean ones.

Overall, our understanding of the relative merits of different cloth mask designs and materials is still limited. The silk head covering with cotton sewn over mouth and nose used one hundred years ago by Wu Lien Teh (4) aligns with recent findings on the use of silk-cotton combinations (93) and approaches to avoid lateral and brow jets $(83,85)$. Wu also noted the potential of improving fit by using a rubber overlay, which has also been rediscovered recently (95). However, there are no modern studies of the efficacy of a full range of mask designs and material combinations, using the most relevant flow rates (at rest or low exertion rate of $15 \mathrm{~L} / \mathrm{min}$ ), and contexts (exhalation from a real person, or simulation using a manikin). Novel approaches to materials, such as using two enveloped layers of paper towel aligned at right angles (146), paper towel combined with a face shield (147), and PVDF nanofibers (148) have not been well studied in the English language literature.

\section{Conclusion}

Our review of the literature offers evidence in favor of widespread mask use as source control to reduce community transmission: non-medical masks use materials that obstruct particles of the necessary size; people are most infectious in the initial period post-infection, where it is common to have few or no symptoms (46-51, 149); non-medical masks have been effective in reducing transmission of respiratory viruses; and places and time periods where mask usage is required or widespread have shown substantially lower community transmission.

The available evidence suggests that near-universal adoption of non-medical masks when out in public, in combination with complementary public health measures could successfully reduce $R_{e}$ (effective-R) to below 1 , thereby reducing community spread if such measures are sustained. Economic analysis suggests that mask wearing mandates could add one trillion dollars to the US $\operatorname{GDP}(34,36)$.

Based on the evidence available, it appears that the positive impact of public mask wearing is, in the words of the precautionary principle "scientifically plausible but uncertain." This notion is reflected in Figure 1 - while researchers may reasonably disagree on the magnitude of transmissibility reduction and compliance, seemingly modest benefits can be highly beneficial in the aggregate. Therefore, widespread use of masks in the community should be utilised (150). 
Models suggest that public mask wearing is most effective at reducing spread of the virus when compliance is high (40). We recommend that mask use requirements are implemented by governments, or when governments do not, by organizations that provide public-facing services. Such mandates must be accompanied by measures to ensure access to masks, possibly including distribution and rationing mechanisms so that they do not become discriminatory. Given the value of the source control principle, especially for presymptomatic people, it is not sufficient for only employees to wear masks, customers must wear masks as well.

It is also important for health authorities to provide clear guidelines for the production, use and sanitization or re-use of face masks, and consider their distribution as shortages allow. Clear and implementable guidelines can help increase compliance, and bring communities closer to the goal of reducing and ultimately stopping the spread of COVID-19.

When used in conjunction with widespread testing, contact tracing, quarantining of anyone that may be infected, hand washing, and physical distancing, face masks are a valuable tool to reduce community transmission. All of these measures, through their effect on $R_{e}$, have the potential to reduce the number of infections. As governments exit lockdowns, keeping transmissions low enough to preserve health care capacity will be critical until a vaccine can be developed.

\section{Materials and Methods}

This is a narrative review of mask use by the public as source control for COVID-19. Using a narrative review as method allows an interdisciplinary approach to evidence synthesis which can deepen understanding and provide interpretation (29). In the context of an evolving novel global pandemic, broadening the evidence base provides a key contribution. Following a literature search of standard indexes, as well as preprint servers, we complemented this with a community-driven approach to identify additional articles, in which researchers suggested related papers, tracked using a publicly available collaborative document. A multidisciplinary team of researchers reviewed, synthesized and interpreted this evidence base. All data underlying the results are available as part of the article and no additional source data are required for interpretation. The working document was uploaded as a preprint in preprints.org and improvements incorporating additional evidence were added. The changes are publicly available (151).

\section{Acknowledgments}

Thank you: Sylvain Gugger ( $\left.\mathrm{AT}_{\mathrm{E}}^{\mathrm{X}} \mathrm{X}\right)$, Luraine Kimmerle (bibtex citations), Linsey Marr (aerosol science), Jon Schwabish (visualization), our reviewers and editor (suggestions and comments), and Koen Vanden Driessche and many others (preprint feedback).

1 Q Wang, C Yu, Letter to editor: Role of masks/respirator protection against 2019-novel coronavirus (COVID-19). Infect. Control. \& Hosp. Epidemiol. 1, 1-7 (2020).

$2 \mathrm{~S}$ Feng, et al., Rational use of face masks in the COVID-19 pandemic. The Lancet Respir. Medicine 0 (2020).

3 LG Goh, T Ho, KH Phua, Wisdom and western science: The work of dr wu lien-teh. Asia Pac. J. Public Heal. 1, 99-109 (1987).

4 LT Wu, A treatise on pneumonic plague. A Treatise on Pneumonic Plague. 4, 373-398 (1926)

5 BJ Cowling, et al., Impact assessment of non-pharmaceutical interventions against coronavirus disease 2019 and influenza in hong kong: an observational study. The Lancet Public Heal. 1 (2020).

6 What countries require masks in public or recommend masks? (https://masks4all.co/ what-countries-require-masks-in-public/) (05) (Accessed on 06/26/2020).
7 JP Higgins, et al., Cochrane handbook for systematic reviews of interventions. (John Wiley \& Sons), (2019)

8 WHO, Handbook for guideline development (2010).

9 T Greenhalgh, Face coverings for the public: Laying straw men to rest. J. Eval. Clin. Pract. 1, e13415 (2020).

$10 \mathrm{Y}$ Wang, et al., Reduction of secondary transmission of sars-cov-2 in households by face mask use, disinfection and social distancing: a cohort study in beijing, china. BMJ Glob. Heal. 5, e002794 (2020)

11 DK Chu, et al., Physical distancing, face masks, and eye protection to prevent person-toperson transmission of sars-cov-2 and covid-19: a systematic review and meta-analysis. The Lancet 1 (2020)

12 P Tuan, et al., Sars transmission in vietnam outside of the health-care setting Epidemiol. \& Infect. 135, 392-401 (2007)

$13 \mathrm{~J} \mathrm{Wu}$, et al., Risk factors for sars among persons without known contact with sars patients, beijing, china. Emerg. infectious diseases 10, 210 (2004).

14 CR Maclntyre, et al., The First Randomized, Controlled Clinical Trial of Mask Use in Households to Prevent Respiratory Virus Transmission. Int. J. Infect. Dis. 12, e328 (2008).

$15 \mathrm{~T}$ Jefferson, et al., Physical interventions to interrupt or reduce the spread of respiratory viruses. Cochrane Database Syst. Rev. 7, CD006207 (2011).

$16 \mathrm{~T}$ Jefferson, et al., Physical interventions to interrupt or reduce the spread of respiratory viruses. Part 1 - Face masks, eye protection and person distancing: systematic review and meta-analysis. medRxiv 1, 2020.03.30.20047217 (2020).

17 CR Maclntyre, AA Chughtai, A rapid systematic review of the efficacy of face masks and respirators against coronaviruses and other respiratory transmissible viruses for the community, healthcare workers and sick patients. Int. J. Nurs. Stud. 1, 103629 (2020).

18 UIU of Edinburgh, Summary: Does the use of face masks in the general population make a difference to spread of infection? (https://www.ed.ac.uk/files/atoms/files/uncover_003-03 summary_-_facemasks_community_anon.pdf) (2020).

19 M GUPTA, K Gupta, S Gupta, The use of facemasks by the general population to prevent transmission of Covid 19 infection: A systematic review. medRxiv 1 (2020).

20 JS Brainard, N Jones, I Lake, L Hooper, P Hunter, Facemasks and similar barriers to prevent respiratory illness such as COVID-19: A rapid systematic review. medRxiv 1 (2020).

21 M Biggerstaff, S Cauchemez, C Reed, M Gambhir, L Finelli, Estimates of the reproduction number for seasonal, pandemic, and zoonotic influenza: a systematic review of the literature. BMC infectious diseases 14, 480 (2014).

$22 \mathrm{~W} \mathrm{He}, \mathrm{GY}$ Yi, Y Zhu, Estimation of the basic reproduction number, average incubation time, asymptomatic infection rate, and case fatality rate for covid-19: Meta-analysis and sensitivity analysis. J. Med. Virol. 1 (2020).

23 T Suess, et al., The role of facemasks and hand hygiene in the prevention of influenza transmission in households: results from a cluster randomised trial; Berlin, Germany, 2009-2011. BMC infectious diseases 12, 26 (2012).

24 BJ Cowling, et al., Facemasks and hand hygiene to prevent influenza transmission in households: a cluster randomized trial. Annals Intern. Medicine 151, 437-446 (2009).

25 AE Aiello, et al., Mask use, hand hygiene, and seasonal influenza-like illness among young adults: a randomized intervention trial. The J. Infect. Dis. 201, 491-498 (2010).

26 AE Aiello, et al., Facemasks, Hand Hygiene, and Influenza among Young Adults: A Randomized Intervention Trial. PLoS ONE 7 (2012).

27 CR Maclntyre, et al., A cluster randomised trial of cloth masks compared with medical masks in healthcare workers. BMJ Open 5, e006577 (2015).

28 D Ogilvie, et al., Using natural experimental studies to guide public health action: turning the evidence-based medicine paradigm on its head. J Epidemiol Community Heal. 74, 203-208 (2020).

$29 \mathrm{~T}$ Greenhalgh, S Thorne, K Malterud, Time to challenge the spurious hierarchy of systematic over narrative reviews? Eur. journal clinical investigation 48 (2018).

30 WC on the Ethics of Scientific Knowledge, Technology, The precautionary principle (2005).

31 CT Leffler, et al., Association of country-wide coronavirus mortality with demographics, testing, lockdowns, and public wearing of masks. The Am. J. Trop. Medicine Hyg. 1 (2020).

32 C Kenyon, Widespread use of face masks in public may slow the spread of SARS CoV-2: an ecological study. medRxiv 1, 2020.03.31.20048652 (2020)

33 W Lyu, GL Wehby, Community use of face masks and covid-19: Evidence from a natural experiment of state mandates in the us: Study examines impact on covid-19 growth rates associated with state government mandates requiring face mask use in public. Heal. Aff. 1, 10-1377 (2020)

34 J Hatzius, D Struyven, I Rosenbery, Face masks and gdp (https://www.goldmansachs.com/ insights/pages/face-masks-and-gdp.html) (2020) (Accessed on 07/03/2020)

$35 \mathrm{SH}$ Wong, et al., Covid-19 and public interest in face mask use. Am. J. Respir. Critical Care Medicine 1 (2020).

36 J Abaluck, et al., The Case for Universal Cloth Mask Adoption and Policies to Increase Supply of Medical Masks for Health Workers, (Social Science Research Network, Rochester, NY), SSRN Scholarly Paper ID 3567438 (2020).

37 VC Cheng, et al., The role of community-wide wearing of face mask for control of coronavirus disease 2019 (covid-19) epidemic due to sars-cov-2. J. Infect. 1 (2020).

38 RO Stutt, R Retkute, M Bradley, CA Gilligan, J Colvin, A modelling framework to assess the likely effectiveness of facemasks in combination with lock-downin managing the covid-19 pandemic. Proc. Royal Soc. A 476, 20200376 (2020)

39 D Kai, GP Goldstein, A Morgunov, V Nangalia, A Rotkirch, Universal masking is urgent in the covid-19 pandemic: Seir and agent based models, empirical validation, policy recommendaions. arXiv preprint arXiv:2004.13553 1 (2020).

$40 \mathrm{~L}$ Tian, et al., Calibrated intervention and containment of the covid-19 pandemic (2020).

$41 \mathrm{~N}$ Ferguson, et al., Report 9: Impact of non-pharmaceutical interventions (npis) to reduce covid19 mortality and healthcare demand (2020)

$42 \mathrm{CN}$ Ngonghala, et al., Mathematical assessment of the impact of non-pharmaceutical interventions on curtailing the 2019 novel coronavirus. Math. Biosci. 1, 108364 (2020).

$43 \mathrm{~J}$ Yan, S Guha, P Hariharan, M Myers, Modeling the Effectiveness of Respiratory Protective 
Devices in Reducing Influenza Outbreak. Risk Analysis 39, 647-661 (2019).

44 DN Fisman, AL Greer, AR Tuite, Brief research report: Bidirectional impact of imperfect mask use on reproduction number of covid-19: A next generation matrix approach. Infect. Dis. Model. 1 (2020).

45 SA Lauer, et al., The Incubation Period of Coronavirus Disease 2019 (COVID-19) From Publicly Reported Confirmed Cases: Estimation and Application. Annals Intern. Medicine 1 (2020)

$46 \mathrm{KKW}$ To, et al., Temporal profiles of viral load in posterior oropharyngeal saliva samples and serum antibody responses during infection by SARS-CoV-2: an observational cohort study. Lancet Infect. Dis. 0 (2020).

47 L Zou, et al., SARS-CoV-2 Viral Load in Upper Respiratory Specimens of Infected Patients. New Engl. J. Medicine 382, 1177-1179 (2020).

48 Y Bai, et al., Presumed asymptomatic carrier transmission of covid-19. JAMA 1 (2020).

$49 \mathrm{~J}$ Zhang, et al., Evolving epidemiology and transmission dynamics of coronavirus disease 2019 outside Hubei province, China: a descriptive and modelling study. The Lancet Infect. Dis. 0 (2020).

50 WE Wei, Presymptomatic Transmission of SARS-CoV-2 â Singapore, January 23âMarch 16 2020. MMWR. Morb. Mortal. Wkly. Rep. 69 (2020).

51 R Wölfel, et al., Virological assessment of hospitalized patients with covid-2019. Nature 1, 1-10 (2020).

52 DP Oran, EJ Topol, Prevalence of asymptomatic sars-cov-2 infection: A narrative review. Annals Intern. Medicine $\mathbf{1}$ (2020).

53 TC Jones, et al., An analysis of sars-cov-2 viral load by patient age. medRxiv 1 (2020).

$54 \mathrm{M} \mathrm{Han}$, et al., Viral rna load in mildly symptomatic and asymptomatic children with covid-19, seoul. Emerg. Infect. Dis. 1 (2020).

55 L Bourouiba, Turbulent Gas Clouds and Respiratory Pathogen Emissions: Potential Implications for Reducing Transmission of COVID-19. JAMA 1 (2020).

56 DK Milton, A rosetta stone for understanding infectious drops and aerosols. J. Pediatr. Infect. Dis. Soc. 9, 413-415 (2020).

57 S Asadi, et al., Aerosol emission and superemission during human speech increase with voice loudness. Sci. reports 9, 1-10 (2019)

58 V Stadnytskyi, CE Bax, A Bax, P Anfinrud, The airborne lifetime of small speech droplets and their potential importance in sars-cov-2 transmission. Proc. Natl. Acad. Sci. 117, 1187511877 (2020).

59 W Wells, On air-borne infection: Study ii. droplets and droplet nuclei. Am. journal Epidemiol. 20, 611-618 (1934)

60 RS Papineni, FS Rosenthal, The size distribution of droplets in the exhaled breath of healthy human subjects. J. Aerosol Medicine 10, 105-116 (1997).

$61 \mathrm{~J}$ Duguid, The size and the duration of air-carriage of respiratory droplets and droplet-nuclei. Epidemiol. \& Infect. 44, 471-479 (1946).

62 L Morawska, et al., Size distribution and sites of origin of droplets expelled from the human respiratory tract during expiratory activities. J. Aerosol Sci. 40, 256-269 (2009).

63 P Anfinrud, V Stadnytskyi, CE Bax, A Bax, Visualizing speech-generated oral fluid droplets with laser light scattering. New Engl. J. Medicine 1 (2020).

64 V Vuorinen, et al., Modelling aerosol transport and virus exposure with numerical simulations in relation to sars-cov-2 transmission by inhalation indoors. Saf. Sci. 130, 104866 (2020).

65 KA Prather, CC Wang, RT Schooley, Reducing transmission of sars-cov-2. Science $\mathbf{1}$ (2020).

$66 \mathrm{~J}$ Gralton, E Tovey, ML McLaws, WD Rawlinson, The role of particle size in aerosolised pathogen transmission: a review. J. Infect. 62, 1-13 (2011).

67 MP Atkinson, LM Wein, Quantifying the routes of transmission for pandemic influenza. Bull. mathematical biology 70, 820-867 (2008).

$68 \mathrm{~J} \mathrm{Ma}$, et al., Exhaled breath is a significant source of sars-cov-2 emission. medRxiv 1 (2020).

$69 \mathrm{~J}$ Yan, et al., Infectious virus in exhaled breath of symptomatic seasonal influenza cases from a college community. Proc. Natl. Acad. Sci. 115, 1081-1086 (2018).

70 Brosseau, N95 Respirators and Surgical Masks | | Blogs | CDC (2009).

$71 \mathrm{NH}$ Leung, et al., Respiratory virus shedding in exhaled breath and efficacy of face masks. Nat. Medicine 1, 1-5 (2020).

72 V Greene, D Vesley, Method for evaluating effectiveness of surgical masks. J. Bacteriol. 83, 663-667 (1962).

73 LB Quesnel, The efficiency of surgical masks of varying design and composition. Br. J. Surg. 62, 936-940 (1975)

74 DF Johnson, JD Druce, C Birch, ML Grayson, A quantitative assessment of the efficacy of surgical and N95 masks to filter influenza virus in patients with acute influenza infection. Clin. Infect. Dis. An Off. Publ. Infect. Dis. Soc. Am. 49, 275-277 (2009).

75 DK Milton, MP Fabian, BJ Cowling, ML Grantham, JJ McDevitt, Influenza Virus Aerosols in Human Exhaled Breath: Particle Size, Culturability, and Effect of Surgical Masks. PLOS Pathog. 9, e1003205 (2013).

$76 \mathrm{KV}$ Driessche, et al., Surgical masks reduce airborne spread of pseudomonas aeruginosa in colonized patients with cystic fibrosis. Am. J. Respir. Critical Care Medicine 192, 897-899 (2015) PMID: 26426786

$77 \mathrm{ME}$ Wood, et al., Face masks and cough etiquette reduce the cough aerosol concentration of pseudomonas aeruginosa in people with cystic fibrosis. Am. J. Respir. Critical Care Medicine 197, 348-355 (2018) PMID: 28930641

78 RE Stockwell, et al., Face masks reduce the release of pseudomonas aeruginosa cough aerosols when worn for clinically relevant periods. Am. J. Respir. Critical Care Medicine 198, 1339-1342 (2018) PMID: 30028634

79 AS Dharmadhikari, et al., Surgical face masks worn by patients with multidrug-resistant tuberculosis: impact on infectivity of air on a hospital ward. Am. journal respiratory critical care medicine 185, 1104-1109 (2012).

80 JFW Chan, et al., Surgical mask partition reduces the risk of non-contact transmission in a golden syrian hamster model for coronavirus disease 2019 (covid-19). Clin. Infect. Dis. 1 (2020).

81 A Davies, et al., Testing the Efficacy of Homemade Masks: Would They Protect in an Influenza Pandemic? Disaster Medicine Public Heal. Prep. 7, 413-418 (2013).
82 Mvd Sande, P Teunis, R Sabel, Professional and Home-Made Face Masks Reduce Exposure to Respiratory Infections among the General Population. PLOS ONE 3, e2618 (2008).

83 I Viola, et al., Face coverings, aerosol dispersion and mitigation of virus transmission risk arXiv preprint arXiv:2005.10720 1 (2020).

$84 \mathrm{~V}$ Kumar, et al., On the utility of cloth facemasks for controlling ejecta during respiratory events. arXiv preprint arXiv:2005.03444 1 (2020).

$85 \mathrm{~S}$ Verma, M Dhanak, J Frankenfield, Visualizing the effectiveness of face masks in obstructing respiratory jets. Phys. Fluids 32, 061708 (2020).

$86 \mathrm{CDC}$, How coronavirus spreads (https://www.cdc.gov/coronavirus/2019-ncov/ prevent-getting-sick/how-covid-spreads.html) (2020) (Accessed on 07/05/2020).

87 E Goldman, Exaggerated risk of transmission of COVID-19 by fomites. The Lancet Infect. Dis. 1 (2020).

88 M Ippolito, P Iozzo, C Gregoretti, G Grasselli, A Cortegiani, Facepiece filtering respirators with exhalation valve should not be used in the community to limit sars-cov-2 diffusion. Infect. Control. \& Hosp. Epidemiol. 1, 1-4 (2020).

$89 \mathrm{NIOSH}, \mathrm{Cdc}$ - niosh publications and products - niosh guide to the selection and use of particulate respirators certified under 42 cfr 84 (96-101) (https://www.cdc.gov/niosh/docs/96-101/ default.html) (1996) (Accessed on 07/07/2020).

$90 \mathrm{H}$ Jung, et al., Comparison of filtration efficiency and pressure drop in anti-yellow sand masks, quarantine masks, medical masks, general masks, and handkerchiefs. Aerosol Air Qual. Res. 14, 991-1002 (2013).

91 WH Organization, , et al., Advice on the use of masks in the context of covid-19: interim guidance, 5 june 2020, (World Health Organization), Technical report (2020).

$92 \mathrm{M}$ Zhao, et al., Household materials selection for homemade cloth face coverings and their filtration efficiency enhancement with triboelectric charging. Nano Lett. 1 (2020).

93 A Konda, et al., Aerosol filtration efficiency of common fabrics used in respiratory cloth masks. ACS Nano 14, 6339-6347 (2020).

94 VM Dato, D Hostler, ME Hahn, Simple Respiratory Mask. Emerg. Infect. Dis. 12, 1033-1034 (2006)

95 DP Runde, et al., The "double eights mask brace" improves the fit and protection and protection of a basic surgical mask amidst covid-19 pandemic. medRxiv 1 (2020).

$96 \mathrm{M}$ van der Sande, $\mathrm{P}$ Teunis, R Sabel, Professional and Home-Made Face Masks Reduce Exposure to Respiratory Infections among the General Population. PLoS ONE 3 (2008).

$97 \mathrm{~V}$ Offeddu, CF Yung, MSF Low, CC Tam, Effectiveness of masks and respirators agains respiratory infections in healthcare workers: A systematic review and meta-analysis. Clin. Infect. Dis. 65, 1934-1942 (2017).

98 AR Wilkes, JE Benbough, SE Speight, M Harmer, The bacterial and viral filtration performance of breathing system filters*. Anaesthesia 55, 458-465 (2000).

$99 \mathrm{Y}$ Long, et al., Effectiveness of N95 respirators versus surgical masks against influenza: A systematic review and meta-analysis. J. Evidence-Based Medicine $\mathbf{n} / \mathbf{a}(2020)$

100 LJ Radonovich, et al., N95 Respirators vs Medical Masks for Preventing Influenza Among Health Care Personnel: A Randomized Clinical Trial. JAMA 322, 824-833 (2019).

101 B Bean, et al., Survival of influenza viruses on environmental surfaces. J. Infect. Dis. 146 47-51 (1982).

102 LM Brosseau, ScD, M Sietsema, P| Apr 01, 2020, COMMENTARY: Masks-for-all for COVID19 not based on sound data (2020).

103 MM Cassell, DT Halperin, JD Shelton, D Stanton, Risk compensation: the achilles' heel o innovations in hiv prevention? BMJ 332, 605-607 (2006).

104 D Rojas Castro, RM Delabre, JM Molina, Give prep a chance: moving on from the risk compensation concept. J. Int. AIDS Soc. 22, e25351 (2019)

$105 \mathrm{JV}$ Ouellet, Helmet use and risk compensation in motorcycle accidents. Traffic injury prevention 12, 71-81 (2011).

106 DJ Houston, LE Richardson, Risk compensation or risk reduction? seatbelts, state laws, and traffic fatalities. Soc. Sci. Q. 88, 913-936 (2007)

107 MD Scott, et al., Testing the risk compensation hypothesis for safety helmets in alpine skiing and snowboarding. Inj. Prev. 13, 173-177 (2007).

$108 \mathrm{Y}$ Peng, et al., Universal motorcycle helmet laws to reduce injuries: a community guide systematic review. Am. journal preventive medicine 52, 820-832 (2017).

109 G Ruedl, M Kopp, M Burtscher, Does risk compensation undo the protection of ski helmet use? Epidemiology 23, 936-937 (2012).

110 B Pless, Risk compensation: Revisited and rebutted. Safety 2, 16 (2016).

111 A Burgess, M Horii, Risk, ritual and health responsibilisation: Japans safety blanketof surgical face mask-wearing. Sociol. health \& illness 34, 1184-1198 (2012).

$112 \mathrm{C}$ Betsch, et al., Social and behavioral consequences of mask policies during the COVID-19 pandemic. Proc. Natl. Acad. Sci. United States Am. 117, 21851-21853 (2020).

113 Imperial College London, Face mask use: Associations with other non-pharmaceutical intervention behaviours (2020).

114 YJ Chen, et al., Comparison of Face-Touching Behaviors Before and During the Coronavirus Disease 2019 Pandemic. JAMA network open 3, e2016924 (2020).

115 G Seres, et al., Face Masks Increase Compliance with Physical Distancing Recommendations During the COVID-19 Pandemic. Work. Pap. 1, 1-39 (2020).

116 M Marchiori, Covid-19 and the social distancing paradox: dangers and solutions. arXiv preprint arXiv:2005.12446 1 (2020).

117 G Seres, AH Balleyer, N Cerutti, J Friedrichsen, M Süer, Face Mask Use and Physical Distancing before and after Mandatory Masking: Evidence from Public Waiting Lines. SSRN Electron. J. 1 (2020).

118 G Joachim, S Acorn, Stigma of visible and invisible chronic conditions. J. advanced nursing 32, 243-248 (2000).

$119 \mathrm{~K}$ Abney, containing tuberculosis, perpetuating stigma: the materiality of n95 respirator masks. Anthropol. South. Afr. 41, 270-283 (2018)

120 E Buregyeya, et al., Acceptability of masking and patient separation to control nosocomial tuberculosis in uganda: a qualitative study. J. Public Heal. 20, 599-606 (2012).

$121 \mathrm{DK} \mathrm{Li}, \mathrm{R}$ Abdelkader, Coronavirus hate attack: Woman in face mask allegedly assaulted by man who calls her 'diseased'. NBC News 1 (2020). 
122 D Pager, H Shepherd, The sociology of discrimination: Racial discrimination in employment, housing, credit, and consumer markets. Annu. Rev. Sociol 34, 181-209 (2008).

$123 \mathrm{C}$ Fernando Alfonso lii, Why some people of color say they won't wear homemade masks (2020) [Online; accessed 9. Apr. 2020].

$124 \mathrm{~T}$ Jan, Two black men say they were kicked out of Walmart for wearing protective masks. Others worry it will happen to them. Wash. Post $\mathbf{1}$ (2020).

$125 \mathrm{~K}$ Wells, Why cant I get tested? Atlantic $\mathbf{1}(2020)$.

126 RE Watson-Jones, CH Legare, The social functions of group rituals. Curr. Dir. Psychol. Sci. 25, 42-46 (2016).

127 R BliegeBird, et al., Signaling theory, strategic interaction, and symbolic capital. Curr. anthropology 46, 221-248 (2005).

128 R Van Houten, L Malenfant, B Huitema, R Blomberg, Effects of high-visibility enforcement on driver compliance with pedestrian yield right-of-way laws. Transp. research record 2393, 41-49 (2013).

129 W Van Damme, W Van Lerberghe, Editorial: Epidemics and fear. Trop. Med. Int. Heal. 5, 511-514 (2000).

130 MA Riva, M Benedetti, G Cesana, Pandemic fear and literature: observations from jack londons the scarlet plague. Emerg. infectious diseases 20, 1753 (2014).

131 E Taal, JJ Rasker, ER Seydel, O Wiegman, Health status, adherence with health recommendations, self-efficacy and social support in patients with rheumatoid arthritis. Patient education counseling 20,63-76 (1993).

132 P Illingworth, WE Parmet, Solidarity and health: A public goods justification. Diametros $\mathbf{4 3}$ 65-71 (2015).

133 LC Chen, TG Evans, RA Cash, , et al., Health as a global public good. Glob. public goods 1 , 284-304 (1999).

134 KK Cheng, TH Lam, CC Leung, Wearing face masks in the community during the covid-19 pandemic: altruism and solidarity. The Lancet 1 (2020).

135 HM Van Der Westhuizen, K Kotze, S Tonkin-crine, N Gobat, T Greenhalgh, Face coverings for COVID-19 : from medical intervention to social practice. Authorea Prepr. 1, 1-7 (2020).

136 The Lancet, COVID-19: protecting health-care workers. The Lancet 395, 922 (2020).

137 JJ Bartoszko, MAM Farooqi, W Alhazzani, M Loeb, Medical masks vs n95 respirators for preventing covid-19 in health care workers a systematic review and meta-analysis of randomized trials. Influ. Other Respir. Viruses $\mathbf{1}$ (2020).

$138 \mathrm{P}$ de Man, et al., Sterilization of disposable face masks by means of standardized dry and steam sterilization processes: an alternative in the fight against mask shortages due to COVID-19 (2020).

139 EN Perencevich, DJ Diekema, MB Edmond, Moving personal protective equipment into the community: Face shields and containment of covid-19. JAMA 1 (2020).

140 EL Larson, et al., Impact of Non-Pharmaceutical Interventions on URIs and Influenza in Crowded, Urban Households. Public Heal. Reports 125, 178-191 (2010).

141 GM Leung, et al., A tale of two cities: community psychobehavioral surveillance and related impact on outbreak control in hong kong and singapore during the severe acute respiratory syndrome epidemic. Infect. Control. \& Hosp. Epidemiol. 25, 1033-1041 (2004).

142 BJ Cowling, et al., Community psychological and behavioral responses through the first wave of the 2009 influenza a (h1n1) pandemic in hong kong. The J. infectious diseases 202, 867876 (2010)

143 WD Bradford, A Mandich, Some state vaccination laws contribute to greater exemption rates and disease outbreaks in the united states. Heal. Aff. 34, 1383-1390 (2015).

144 SY Del Valle, R Tellier, GS Settles, JW Tang, Can we reduce the spread of influenza in schools with face masks? Am. journal infection control 38, 676-677 (2010).

145 AA Chughtai, et al., Contamination by respiratory viruses on outer surface of medical masks used by hospital healthcare workers. BMC infectious diseases 19, 1-8 (2019).

$146 \mathrm{~K} \mathrm{Kwong,} \mathrm{Hkmask}$ manual (https://diymask.site/) (2020) (Accessed on 07/06/2020).

147 Consumer Council Hong Kong, DIY Face Mask - 8 Steps in Making Protective Gear | Consumer Council (2020) [Online; accessed 8. Apr. 2020].

148 C Akduman, Cellulose acetate and polyvinylidene fluoride nanofiber mats for $\mathrm{n} 95$ respirators. J. Ind. Textiles 1, 1528083719858760 (2019).

$149 \mathrm{~N}$ van Doremalen, et al., Aerosol and Surface Stability of SARS-CoV-2 as Compared with SARS-CoV-1. New Engl. J. Medicine 0, null (2020).

150 T Greenhalgh, MB Schmid, T Czypionka, D Bassler, L Gruer, Face masks for the public during the covid-19 crisis. BMJ 369 (2020).

151 Howard, Face masks against covid-19: An evidence review. preprints.org 1 (2020). 